\title{
Molecular Identification of the Dermatophytes Causing Tinea Diseases Using ITS Sequencing Analysis
}

\author{
Tiba Salim Naseif ${ }^{1}$, Abdulameer Jasim Mohammed ${ }^{2}$, Haael Subhi Abbas ${ }^{3}$ \\ ${ }^{1}$ Microbiologist, Master, Directorate Wasit of Health, Ministry of Health, Iraq, ${ }^{2}$ Asst. Prof., PhD, \\ ${ }^{3}$ Asst. Lec., Master, Collage of Health and Medical Technology, Middle Technical University, Iraq
}

\begin{abstract}
Background: Tinea is a group of keratinophilic fungal infections caused by dermatophytes that invade the skin, hair, and nails. As the conventional methods for dermatophytes detection are time-consuming or lack enough specificity, accurate diagnostic methods such as molecular techniques are required for precise identification and differentiate between two closely related species of dermatophytes.
\end{abstract}

Objective: To disclose the useful role of DNA sequencing in the recognition of dermatophytes using the ITS region and comparing the current local isolates with those provided by the NCBI.

Method: Thirty clinical tinea specimens were collected from the patients between December 2016 and June 2017. All specimens were examined microscopically then cultured on DTM. Positive dermatophytes' cultures were confirmed by PCR using ITS1 and ITS4 primers. The PCR products were sequenced for species-specific dermatophyte identification.

Result: Culture results of 30 specimens revealed predominant positive dermatophytes (23, 77\%) comparing the rest of no growth. The PCR amplification of the 23 dermatophytes for species identification showed distinct fragment lengths for different species; $550 \mathrm{bp}$ for $T$. tonsurans and $T$. equinum, $650 \mathrm{bp}$ for $T$. verrcossum, T. bullosum and M. appendiculatum, 690bp for T. rubrum and T. mentagrophytes, $700 \mathrm{bp}$ for T. interdigitale, and 740bp for M. canis and E. floccosum. Sequencing analysis exhibited a novel local $T$. rubrum strain that recorded in the NCBI with accession number (MG786552.1). The genetic compatibility between the reference strain of T. rubrum in India and the local strain was 99\%.

Conclusion: Dermatophytes identification by sequencing analysis of the ITS region is a faster, precise, and more dependable diagnosis at species and subspecies levels than the conventional laboratory methods.

Keywords: Dermatophyte, PCR, ITS, Sequencing.

\section{Introduction}

Tinea (dermatophytosis) is a mycotic infection brought about by dermatophytes; filamentous fungi invading the keratinized tissues of the skin, hairs, and nails. Dermatophytes are restricted to keratinized layers

\section{Corresponding Author:}

\section{Haael Subhi Abbas}

Asst. Lec., Master, Collage of Health and Medical

Technology, Middle Technical University, Iraq as they have keratinase enzymes and require keratin for growth ${ }^{[1]}$. Dermatophytes affiliated to the genera "Trichophyton, Epidermophyton, and Microsporum", with two possible infections pathways, directly (person to person contact) and/or indirectly (animal to human contact) ${ }^{[2]}$. Tinea, dependent on the affected site, has been clinically classified into "Tinea capitis (head), Tinea barbae (beard), Tinea corporis (body), Tinea manuum (hand), Tinea cruris (groin), Tinea pedis (foot), and Tinea unguium (nail)" "[3]. Direct microscopy of clinical tinea specimens is a speedy method for fungal structures recognition, but it is non-specific and less precise $\mathrm{e}^{[4]}$. 
Cultivated dermatophytes can be pinpointed dependent upon various morphological hallmarks like colony pigmentations, texture, outgrowth rate, and presence of conidia, hence culture is important for both therapeutic and epidemiological studies ${ }^{[5]}$. Besides, the conventional laboratory methods for dermatophytes detection is either slow or lack enough specificity, and in sometimes the cultural features were unpredictable, thus the perfect diagnostic methods for dermatophytosis should be simple, specific, rapid, and cost-effective ${ }^{[6]}$. Molecular methods for discrimination between the genotyping characteristics of the dermatophytes species are more specific, precise, rapid, and differentiate between two closely related species as well as are less likely to be affected by external influences such as temperature and chemotherapy ${ }^{[7]}$. Molecular techniques also provide a better understanding of classification, epidemiology, and ecology. Recently, the diagnosis of dermatophytes dependent on DNA amplification and sequencing analysis of the internal transcribed spacer (ITS) regions ${ }^{[8]}$.

The ITS regions of the ribosomal DNA (rDNA) gene in the dermatophytes were used as a reliable marker for species identification. Also, the ITS region is a chunk of unusable RNA existing amidst formational ribosomal RNA (rRNA) on the universal predecessor transcript ${ }^{[9]}$. The ITS region is highly changeable even between closely related species of dermatophytes and the sequence consistency of its region is vastly utilized in taxonomy and molecular phylogeny as it can be amplified effortlessly even from little amounts of DNA (as a result of many rRNA genes copies) ${ }^{[10]}$. As mentioned earlier, the current study aimed to discover the beneficial role of DNA sequencing in the recognition of dermatophytes causing tinea diseases utilizing the ITS region and matching the current local isolates with those provided by National Center Biotechnology Information (NCBI) data.

\section{Materials and Method}

Subjects: Twenty-three clinical tinea specimens (skin scrapings, nails and hair clippings) were collected from patients, irrespective of age and gender, attending the Dermatology Department of Kadhimiya Teaching Hospital/Baghdad between December 2016 and June 2017. The clinical identification of tinea was performed by a specialized dermatologist, and the patient's medical history was recorded using a self-administered questionnaire. However, a proper explanation of the study was addressed to the patients and the consent was taken from them before the collection of the sample.

The specimens were examined microscopically after treating with $20 \%$ potassium hydroxide. The specimens were also cultured on Dermatophyte Test Medium (DTM) (HiMedia Laboratories, India) and incubated at $28^{\circ} \mathrm{C}$ for 2-4 weeks. Dermatophytes were disclosed based on a color change in the DTM due to their ability to produce alkali substances that promote the $\mathrm{pH}$ and change the medium's phenol red from yellow to red ${ }^{[11]}$. DNA extraction performed for positive dermatophytes' cultures using Fungal/Bacterial DNA MiniPrepTM kit (Zymo Research, USA), DNA extraction of an appropriate amount of fungal cells by bead bashing method, which depends on disruption of the cell wall by beads according to the manufacturer instructions. Extracted DNAs were electrophoretically on $1 \%$ agarose gel to determine DNA bands through which observed by UV transilluminator. Thereafter, DNAs were amplified by polymerase chain reaction (PCR) using universal primers; a forward primer (ITS1 F: 5'- TCCGTAGGTGAACCTGCGG-3') and a reverse primer (ITS4 R: 5'-TCCTCCGCTTATTGATATGC-3'). Primers set supplied by Integrated DNA Technologies (IDT Company, Canada).The PCR amplification was done using a Thermal Cycler (Gene Amp, PCR system 9700, Applied Biosystems, USA) in a total volume of $25 \mu 1$ consisting of $1.5 \mu 1$ DNA, $5 \mu 1$ Taq PCR PreMix (Intron, Korea), $1 \mu 1$ of each primer and $16.5 \mu$ lof nucleasefree distilled water. Thermal cycling conditions were applied as: Denaturation at $94{ }^{\circ} \mathrm{C}$ for $3 \mathrm{~min}$, followed by 35 cycles of $94{ }^{\circ} \mathrm{C}$ for $45 \mathrm{~s}, 52^{\circ} \mathrm{C}$ for $1 \mathrm{~min}$ and $72{ }^{\circ} \mathrm{C}$ for 1 min with final incubation at $72{ }^{\circ} \mathrm{C}$ for $7 \mathrm{~min}$. The PCR products were analyzed after RedSafe ${ }^{\mathrm{TM}}$ staining by $2 \%$ agarose gel electrophoresis alongside DNA Ladder (100 bp). Sanger sequencing of gene performed by National Instrumentation Center for Environmental Management $(\mathrm{NICEM})^{[12]}$ (biotechnology lab, DNA sequencer 3730XL, Applied Biosystems, USA). A homology search was conducted using the Basic Local Alignment Search Tool (BLAST) program that is available at NCBI (online at https://www.ncbi.nlm.nih.gov) and BioEdit/ MEGA6 program for multiple sequence alignment, and the sequences result compared with NCBI control strains.

\section{Results}

The clinical distribution of the types of tinea was illustrated in Figure (1), with higher prevalence of Tinea 
corporis $(8,26.7 \%)$, Tinea pedis $(7,23.3 \%)$ and Tinea capitis $(6,20.0 \%)$ compared to other types of Tinea among the 30 cases included in this study.

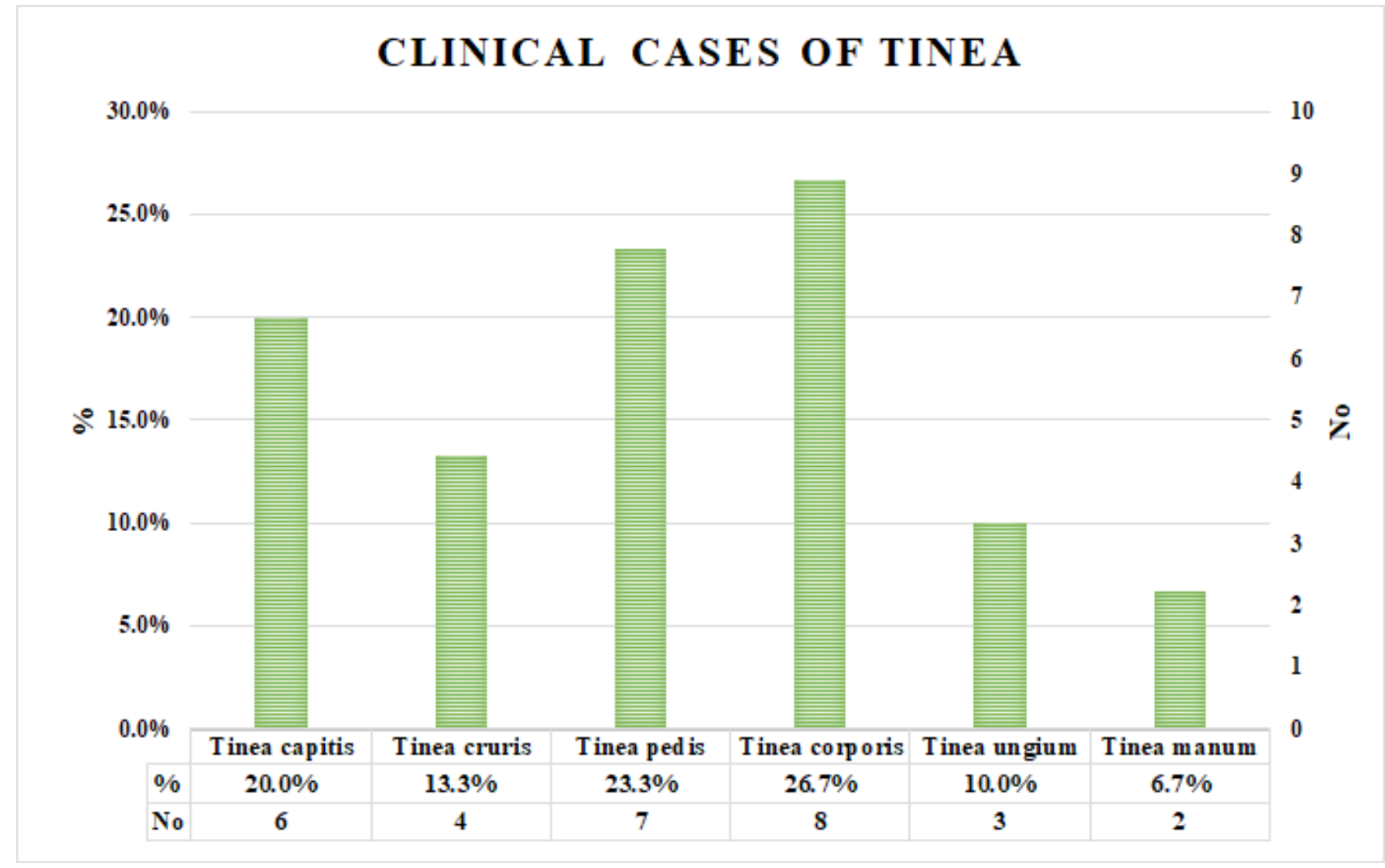

Figure (1): Clinical distribution of Tineatypes among thirty cases in this study.

Figure (2) showed the cultivation results of Tinea cases on DTM. Among 30 clinical cases in this study, 23 (77\%) predominant positive dermatophytes cultures contrasting $7(23 \%)$ of no growth.

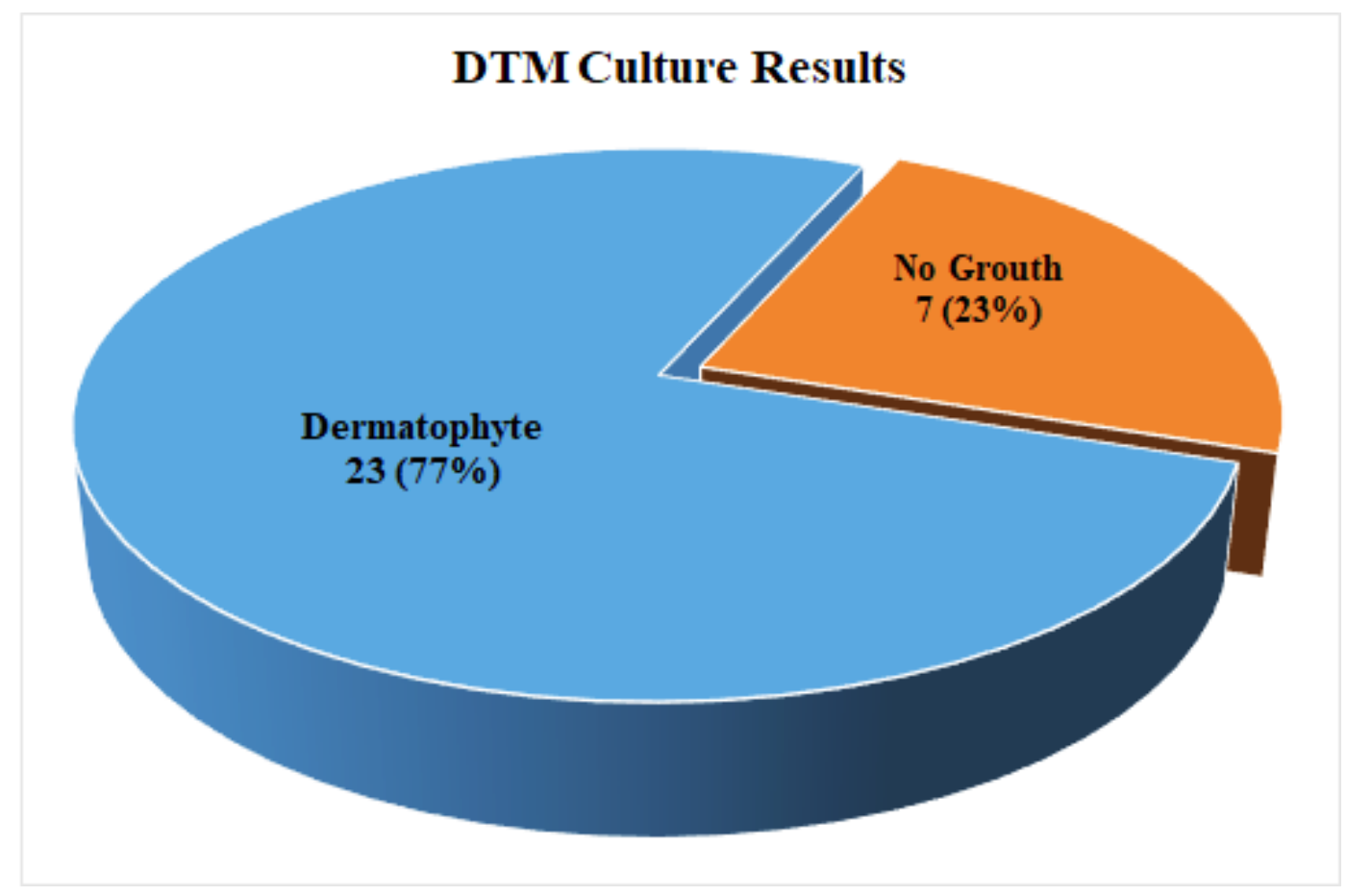

Figure (2): The cultivation results of thirty clinical tinea cases on DTM. 


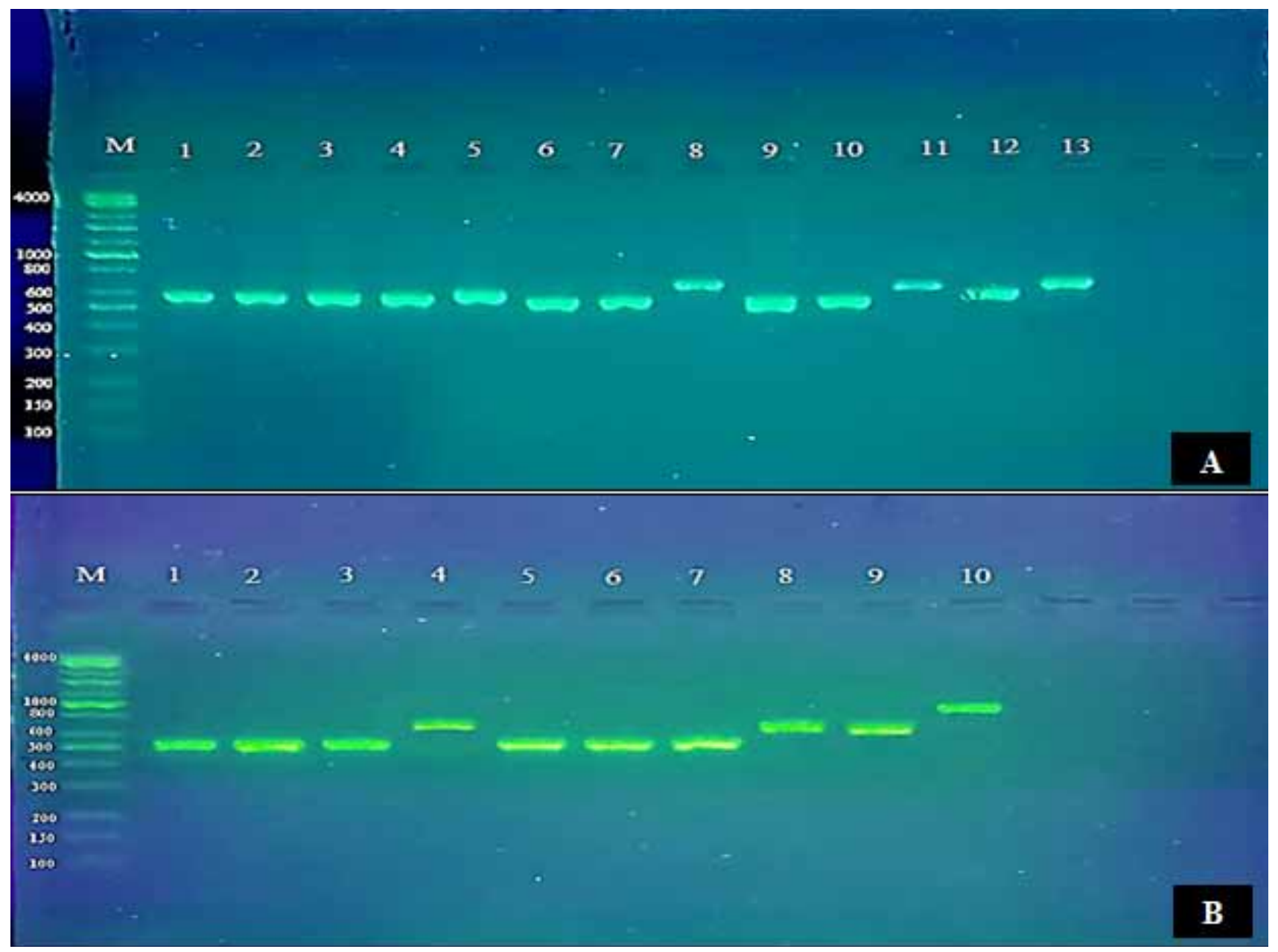

Figure (3): PCR products of ITS region of band size 550-740bp, electrophoresis on $2 \%$ agarose at 70 volt/ $\mathrm{cm}^{2}$ for 1:30 hours with UV visualization. (A) Lane M: DNA ladder (100bp), lanes (1-13) PCR products. (B) Lane M: DNA ladder (100bp), lanes (1-10) PCR products.

DNA sequencing analysis of highly variable ITS regions (ITS1 and ITS2 and the 5.8S gene) was applied for 23 PCR products of dermatophytes isolates. This study revealed a predominant T. Mentegrophytes (22\%) and E. floccosum (17\%) followed by T. tunsurans, T. bullosom, and T. rubrum $(13 \%, 13 \%$, and $9 \%$ respectively), comparing $M$. appendiculatum, $M$. canis, T. equinium, T. interdigitalie, and $T$. verrcossum with lower prevalence ( $4 \%$ for each one). The current study exhibited a novel $T$. rubrum strain that recorded in the NCBI with accession number (MG786552.1) as illustrated in Figure (4). The genetic compatibility between the reference strain of T. rubrum in India and the novel strain in the current study was manifested in Figure (5), through which the compatibility percentage between these two strains was $99 \%$. 
Trichophyton rubrum isolate RM7 internal transcribed spacer 1, partial sequence; $5.8 \mathrm{~S}$ ribosomal RNA gene and internal transcribed spacer 2, complete sequence; and 28S ribosomal RNA gene, partial sequence. Sequence ID: KF437402.1

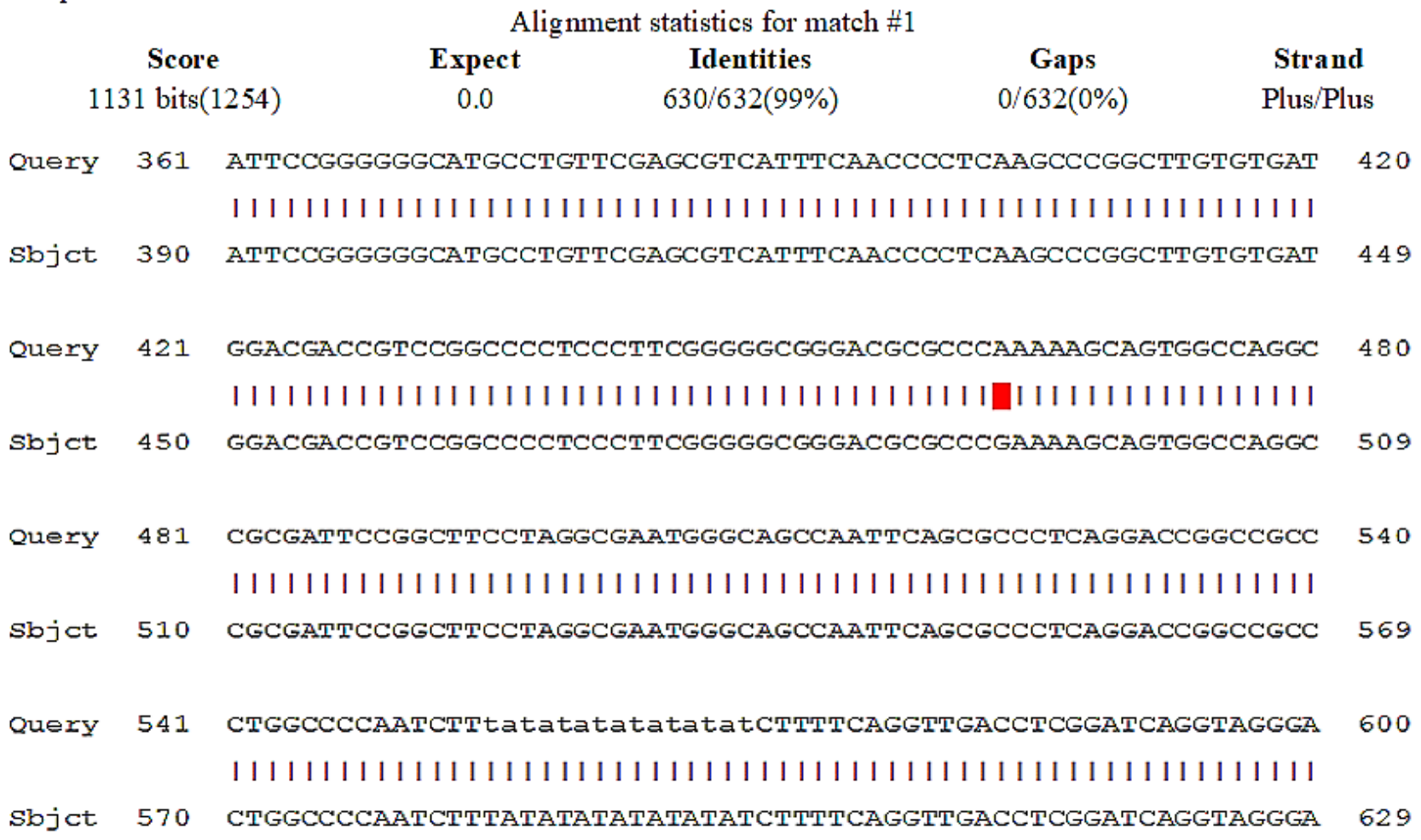

Figure (4): Sequencing analysis of the novel local T. rubrumstrain in Iraq compared with the NCBI.

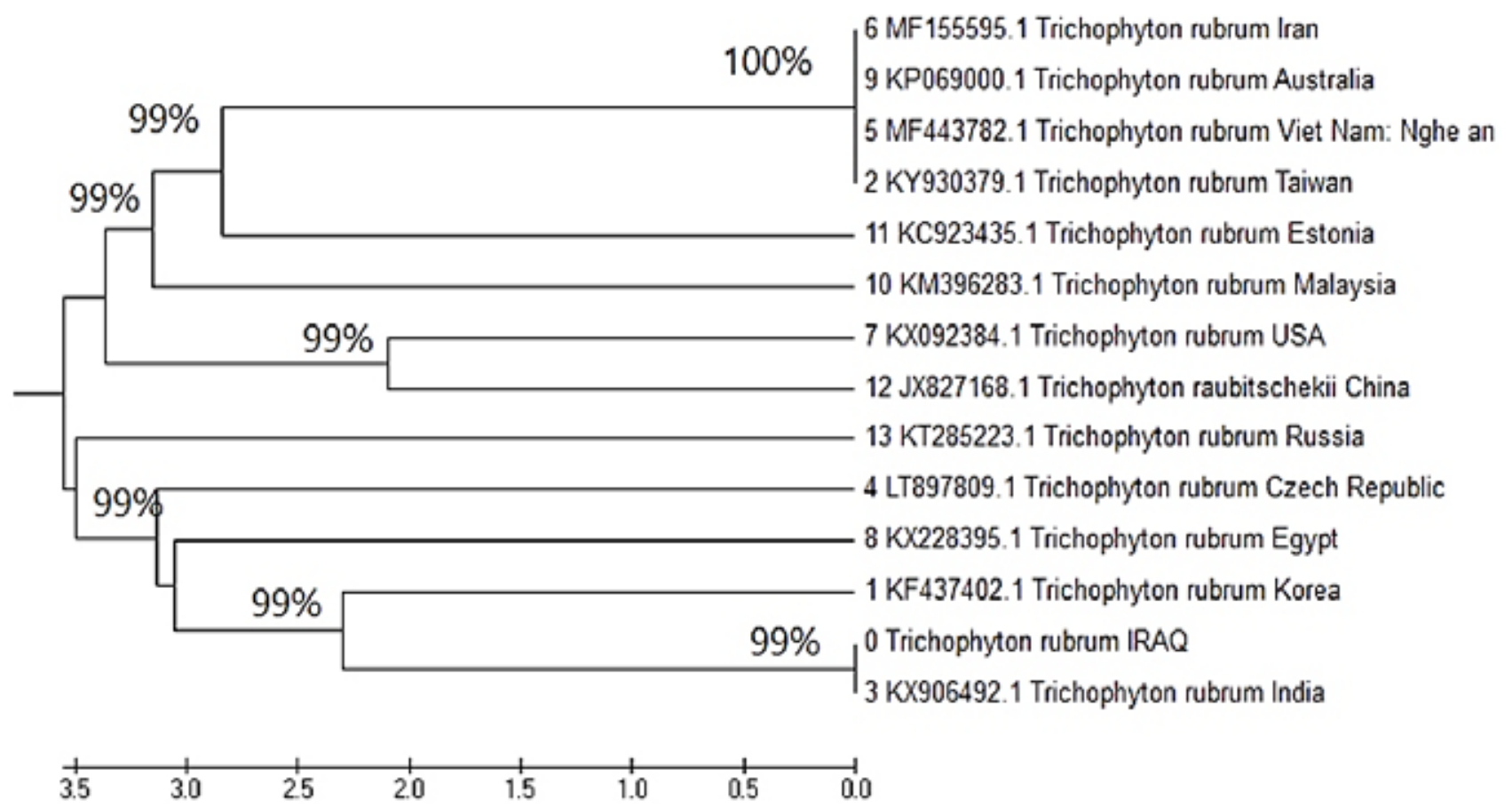

Figure (5): Phylogenetic tree of the novel local T. rubrum strain sequences in Iraq (Mega v.6). 


\section{Discussion}

Identification of dermatophytes at the species level is very important not for diagnosis only but for therapeutic strategies since the conventional diagnostic procedures for dermatophytes detection are slow, lower specificity, and further testing was required to confirm their identity. Misidentification also was one of the main reasons that make the dermatophytes among the first fungal groups studied using molecular techniques.

In this study, thirty clinically diagnosed specimens of patients with different types of tinea infections were collected and cultivated on DTM as differential and selective medium for dermatophytes. The current findings demonstrated that 23 among 30 clinical specimens were positive (red color change) for dermatophytes. These results were in harmony with a study by Singh et al. in India [13], which revealed that DTM is a well reliable media for eclectic incipient isolation, and early discernment, of dermatophytes. The failure of the growth of 7 specimens on DTM might be due to the utilization of antifungal or corticosteroid drugs before specimen collection, clinical misdiagnosis, or inadequate specimen ${ }^{[14]}$. Besides, the vying between the dermatophytes and the escorted saprophytes at the lesion sites may disserve and forbid the growth of the dermatophytes on agar media ${ }^{[15]}$.

Molecular techniques are more advantageous for dermatophyte identification as they are rapid, highly sensitive and specific. Moreover, these methods depend on genetic character, which is more persistent than phenotypic features, and they can identify atypical dermatophytes that could not be recognized by culturebased techniques ${ }^{[16]}$. The PCR technique has rapidly become one of the most widely used techniques in molecular biology for its high discriminatory power and reproducibility, because it is rapid, simple and requires very little specimen materials ${ }^{[17]}$. Twentythree dermatophyte isolates were confirmed using PCR for species identification. In the PCR, the ITS region was amplified using the primers ITS1 and ITS4. As expected, the fragment size of PCR products from different species showed different lengths. The size of the amplified product was approximately $690 \mathrm{bp}$ for $T$. rubrum and T. mentagrophytes, $740 \mathrm{bp}$ for $M$. canis and E. floccosum, 650 bp for $T$. verrcossum, T. bullosum and $M$. appendiculatum, $550 \mathrm{bp}$ for $T$. tonsurans and $T$. equinum, $700 \mathrm{bp}$ for $T$. interdigitale, whereas $770 \mathrm{bp}$ for Microsporum $s p$. These study results were consistent with several studies using PCR as a reliable technique for species-specific identification of dermatophytes, which mentioned that molecular methods utilize ITS regions are more accurate, specific, and effective in the rapid recognition of dermatophyte species in clinical and epidemiological approaches based on the fragment size length ranging from 550 to $740 \mathrm{bp}{ }^{[18,19]}$.

DNA sequencing, in this study, was applied for the PCR products, via the maximization of the ITS region in the rDNA gene. The ITS is a powerful significant marker through which it can manage with phylogenetically congenerical extra far fungal species. The PCR products were sequenced in South Korea (Macrogene lab) as a standard sequencing service. Sequence analysis of the more variable ITS region is the beneficial discernment assay for dermatophytes' species recognition displaying highly pleomorphism of ITS region inside the same genus as well as it's a potential tool to resolve the phylogenetic relationships within the family Arthrodermatacae [20].

The ITS region of the "nuclear ribosomal DNA repeat unit" is frequently sequenced for enquiries of systematics and taxonomy within the genus/species levels. The region $650 \mathrm{bp}$ can be gained via a single run of DNA sequencing and of its sub-regions (ITS1 and ITS2 and 5.8S gene) which manifested a supremely developmental degree of species specificity ${ }^{[21]}$. In current study, the ITS1F and ITS4Rprimers were used to amplify the spacers ITS1 and ITS2 to characterize fungal abundance and diversity.Among the 23 sequenced dermatophytes, a novel T. rubrum strain was identified and recorded in the NCBI with accession number MG786552.1, which was isolated from the groin of an adult male with Tinea cruris that lived in a rural area. As mentioned, the nucleotide sequencing revealed that the genetic variations happened in a single region, with one transition only at the position (491) that convert it from Guanine (G) to Adenine (A).

This study also demonstrated a highly similar percentage (99\%) of genetic affinity between the recorded isolate and the NCBI, and the phylogenetic analysis figured out the high compatibility percentage (about 99\%) between the local novel strain of T. rubrum in Iraq and T. rubrum strain in India. Interpretation of this result, in our opinion, might be due to Indian arrivals to Iraq who had tinea and their admixture with Iraqis resulting in the transmission of tinea infection. Thus, the coinfection of the two distinct strains of T. rubrum leads to genetic mutations with the emergence of a new strains. 


\section{Conclusion}

Dermatophytes identification by sequencing analysis of the ITS region is a faster, precise, and more dependable diagnosis that can replace, with facilities supported, the conventional methods. Also, this technique becomes essential for correct recognition of dermatophyte species for proper therapies, realize the source of infection, and the epidemiology of uncommon dermatophyte species.

Acknowledgement: Our thanks for a dermatologist Dr. Ali Abbas Al-Gburi and the laboratory>s staff in Kadhimiya Teaching Hospital for their assistance.

\section{Conflict of Interest: None}

Source of Findings: None

Ethical Clearance: None

\section{References}

1. Najem MH, Al-Salhi MH, Hamim SS. Study of Dermatophytosis prevalence in Al-Nassiriyah cityIraq. World Journal of Pharmaceutical Sciences. 2016;4(4):166-72.

2. Moriarty B, Hay R, and Morris-Jones R. The diagnosis and management of tinea. British Medical Journal. 2012;345(7865): 37-42.

3. Suganthi M. Pathogenesis and clinical significance of dermatophytes: A comprehensive review. Innovations in Pharmaceuticals and Pharmacotherapy. 2017;5(1):62-70.

4. Garg J, Tilak R, Singh S, Gulati AK, Garg A, Prakash $\mathrm{P}$, and Nath G. Evaluation of pan-dermatophyte nested PCR in diagnosis of onychomycosis. Journal of clinical microbiology. 2007;45(10): 3443-3445.

5. Alzubaidy TS, Mohammed AJ, Al-Gburi AA. Comparison of Two Conventional Method for Identification of Dermatophyte Fungi. Ibn ALHaitham Journal For Pure and Applied Science. 2018 Sep 12;31(2):21-30.

6. Elavarashi E, Kindo AJ, Kalyani J. Optimization of PCR-RFLP Directly from the Skin and Nails in Cases of Dermatophytosis, Targeting the ITS and the 18S Ribosomal DNA Regions. Journal of clinical and diagnostic research. 2013;7(4): 646651.

7. Gräser Y, Scott J, Summerbell R. The new species concept in dermatophytes - a polyphasic approach. Mycopathologia. 2008;166(5-6), 239.
8. Cafarchia C, Iatta R, Latrofa MS, Gräser Y, Otranto D. Molecular epidemiology, phylogeny and evolution of dermatophytes. Infection, Genetics and Evolution. 2013;20:336-51.

9. Ghojoghi A, Falahati M, Abastabar M, Ghasemi Z, Ansari S, Farahyar S, Roudbary M. Molecular identification and epidemiological aspects of dermatophytosis in Tehran, Iran. Research in Molecular Medicine. 2015;3(3):11-6.

10. Schoch CL, Seifert KA, Huhndorf S, Robert V, Spouge JL, Levesque CA, Chen W, Fungal Barcoding Consortium. Nuclear ribosomal internal transcribed spacer (ITS) region as a universal DNA barcode marker for Fungi. Proceedings of the National Academy of Sciences. 2012;109(16): 6241-6.

11. HiMedia Laboratories. D.T.M. Agar Base (Dermatophyte Test Agar Base). [Online] Available at: http://himedialabs.com/TD/M188.pdf.

12. National Instrumentation Center for Environmental Management. [Online]. Available from: https:// nicem.snu.ac.kr/main/?en_skin=index.html.

13. Singh TN, Zamzachin G, Singh NB. Recognition of dermatophytes by dermatophyte test medium. International Journal of Current Microbiology and Applied Sciences. 2016;5(10):1125-9.

14. Munir S, Ganaie F, Kumar B, Tewari R, Badakshaan S. Epidemiologic, Clinico-mycological aspects of fungal infections of skin and its appendages. Journal of Evolution of Medical and Dental Science. 2014;3(16):4212-9.

15. Habeb KA, Maikhan HK, Rachid SK. Molecular Identification of Dermatophytes among Clinical Isolates. Asian Journal of Natural \& Applied Sciences. 2016;5(2):108-118.

16. Ismael HM. PCR based identification of two medically important dermatophyte fungi, Microsporum gypseum and Trichophyton mentagrophytes. American International Journal of Research in Formal, Applied \& Natural Sciences. 2013;4(1):8-11.

17. Jha BK, Murthy SM, Devi NL. Molecular identification of dermatophytosis by polymerase chain reaction (PCR) and detection of source of infection by restricted fragment length polymorphism (RFLP). Journal of College of Medical Sciences-Nepal. 2012;8(4):7-15. 
18. Farokhipor S, Ghiasian SA, Nazeri H, Kord M, Didehdar M. Characterizing the clinical isolates of dermatophytes in Hamadan city, Central west of Iran, using PCR-RLFP method. Journal de mycologie medicale. 2018;28(1):101-5.

19. Verrier J, Monod M. Diagnosis of dermatophytosis using molecular biology. Mycopathologia. 2017; 182(1-2):193-202.
20. Sitterle E, Frealle E, Foulet F, Cabaret O, Cremer G, Guillot J, Delhaes L, Botterel F. Trichophyton bullosum: a new zoonotic dermatophyte species. Medical mycology. 2012;50(3):305-9.

21. Yang RH, Su JH, Shang JJ, Wu YY, Li Y, Bao DP, Yao YJ. Evaluation of the ribosomal DNA internal transcribed spacer (ITS), specifically ITS1 and ITS2, for the analysis of fungal diversity by deep sequencing. PloS one. 2018;13(10):e0206428. 